\title{
Coelidiana ferruginea sp.n. (Hemiptera, Auchenorrhyncha, Neocoelidiinae) de Mato Grosso, Brasil ${ }^{1}$
}

\author{
Larissa De Bortolli Chiamolera ${ }^{2}$ \\ Rodney R. Cavichioli ${ }^{3}$
}

\begin{abstract}
Coelidiana ferruginea sp.n. (Hemiptera, Auchenorrhyncha, Neocoelidiinae) from Mato Grosso, Brazil. A new species of Coelidiana Oman, 1936 is described from Sinop, Mato Grosso, Brazil. The new species can be distinguished by the aspect of the male genitalia, mainly by the aedeagus apex.

KEY WORDS. Hemiptera, Auchenorrhyncha, Neocoelidiinae, Coelidiana, new species
\end{abstract}

OMAN (1936: 397) descreveu Coelidiana (Jassinae), designando Neocoelidia rubrolineata Baker, 1898 como espécie-tipo, do Brasil. Nesse mesmo trabalho transferiu mais quatro espécies para o gênero: Neocoelidia bimaculata Baker, 1898; Neocoelidia modesta Baker, 1898; Neocoelidia inflata Osborn, 1923 e Neocoelidia croceata Osborn, 1923.

Evans (1947) transferiu o gênero Coelidiana da subfamília Jassinae para a subfamília Neocoelidiinae.

DELoNg (1953) descreveu três novas espécies para o gênero: $C$. spina, $C$. bidentata e C.(Nelidina) defila. Descreveu um gênero novo: Acocoelidia para abrigar duas novas espécies: A. unipuncta e A. anomala.

KRAMER (1959) transfere Chinaia undata Linnavouri, 1956 para o gênero Coelidiana.

METCALF (1964) catalogou todas as espécies do gênero que foram descritas até dezembro de 1955.

LINNAVUORI \& HELLER (1961) descreveram mais duas espécies: Coelidiana (Stenocoelidia) flavida e C. (Coelidiana) rotundiceps.

KRAMER (1964) revisou vários gêneros de Neocoelidiinae e sinonimizou Acocoelidia DeLong (1953) para Coelidiana, incluindo A. unipuncta e A. anomala. Além dessa sinonímia faz duas novas combinações: Neocoelidia coronata Ball, 1916 e $N$. croceata Osborn, 1923. Esta última já havia sido transferida para Coelidiana por OMAN (1936). Nesse trabalho Kramer descreve Coelidiana assim: com carena separando a face da coroa; cabeça, incluindo os olhos, mais estreita que o pronoto; antenas tão longas quanto o comprimento do corpo; venação da asas anteriores obscura, exceto apicalmente; coloração em tons de amarelo, com manchas avermelhadas na cabeça, pronoto e escutelo; placa subgenital fusionada em quase

1) Contribuição número 1134 do Departamento de Zoologia, Universidade Federal do Paraná.

2) Departamento de Zoologia, Universidade Federal do Paraná. Caixa Postal 19020, 81531-990 Curitiba, Paraná, Brasil. 
toda a sua extensão; pigóforo variável, sem processos dorsais; conetivo em forma de "Y"; estilos, em vista lateral, curvado apicalmente; edeago simples, fino ou moderadamente robusto e curvado no ápice. Ainda nesse mesmo trabalho eleva o subgênero Nelidina para gênero.

LinNaVUORI (1965) descreveu quatro novas espécies de Coelidiana: $C$. brasiliensis, $C$. distinctissima, $C$. lurida e $C$. signaticeps.

KRAMER (1967) revisou o gênero Coelidiana descrevendo quatro novas espécies: $C$. colens, $C$. ancora, $C$. durata e $C$. patrator e registra quinze espécies para para o gênero, como segue: C. rubrolineata (Baker, 1898); C. bimaculata (Baker, 1898); C. unipuncta (DeLong, 1953); C. anomala (DeLong, 1953); C. spina DeLong, 1953; C. bidentata DeLong, 1953; C. flavida Linnavuori \& Heller, 1961; C. brasiliensis Linnavuori, 1965; C. signaticeps Linnavuori, 1965; C. undata (Linnavuori, 1965); C. colens Kramer, 1967; C. ancora Kramer, 1967; C. durata Kramer, 1967; C. patrator Kramer, 1967; C. croceata (Osborn 1923) e as espécies C. coronata (Ball, 1916), C. distinctissima Linnavuori, 1965 e C. lurida Linnavuori, 1965 são colocadas em posição incerta por terem sido descritas com base em um único espécimen fêmea. No entanto, omite três espécies incluídas anteriormente no gênero: $C$. modesta, $C$. inflata e $C$. rotundiceps.

DeLong \& Kolbe (1975) descreveram mais uma espécie: C. plaga.

Analisando o material da Coleção Entomológica do Departamento de Zoologia da Universidade Federal do Paraná, descobriu-se mais uma espécie a qual é descrita abaixo.

\section{Coelidiana ferruginea sp.n.}

Figs 1-8

Holótipo: BrasiL, Mato Grosso: Sinop, macho, X.1975, M. Alvarenga leg. .

Parátipos. Mesmos dados do holótipo, 9 fêmeas e 2 machos. Tanto o holótipo quanto os parátipos estão depositados na Coleção Pe. Jesus Santiago Moure, Departamento de Zoologia, Universidade Federal do Paraná.

Diagnose. Caracterizada por apresentar edeago não muito delgado, sem processo apical, placa subgenital sem macrocerdas.

Holótipo macho. Medidas (em mm), holótipo/fêmea: comprimento total 8,00/9,75; comprimento mediano da cabeça $0,64 / 0,80$; distância transocular $1,60 / 1,72$; distância interocular $0,84 / 0,94$; comprimento mediano do pronoto $0,64 / 0,80$; distância entre os úmeros $2,08 / 2,34$; largura máxima do escutelo $1,64 / 1,74$; comprimento mediano do escutelo $1,46 / 1,56$; comprimento das tégminas $6,50 / 8,00$; largura máxima das tégminas 1,90/2,33.

Cabeça, em vista dorsal, levemente pronunciada, de comprimento mediano aproximadamente $2 / 5$ da distância transocular e $3 / 4$ da interocular; superfície dorsal lisa e com uma pequena elevação mediana; margem anterior arredondada; com carena na transição entre a coroa e a face; ocelos não evidentes, localizados na face anterior aos lóbulos suprantenais e próximos da margem anterior da coroa; superfície dorsal; lóbulos suprantenais não protuberantes dorsalmente, em vista lateral, 

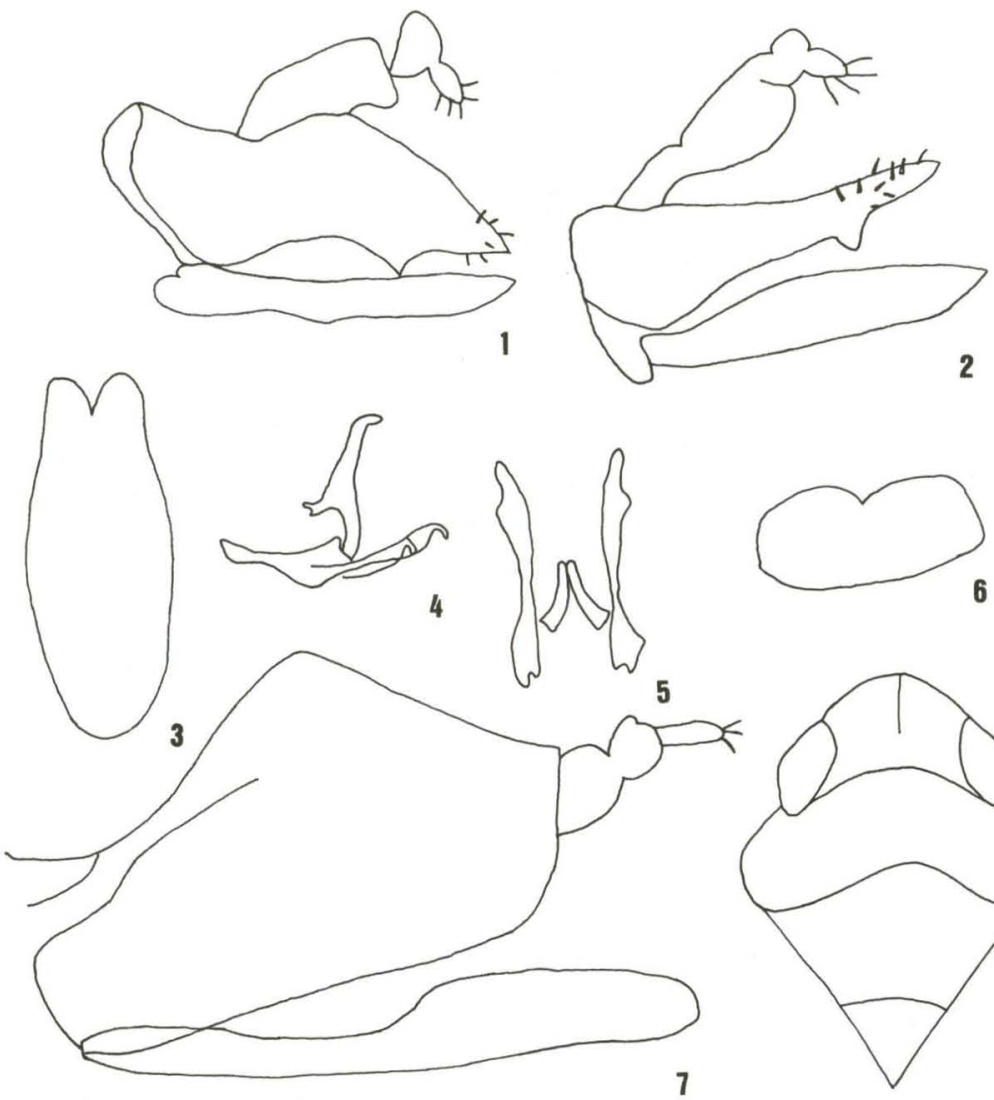

6

Fig. 1-8. Coelidiana ferruginea sp.n.. (1) Cabeça-pronoto-escutelo, vista dorsal; (2) pigóforo, vista lateral; (3) placa subgenital, vista ventral; (4) estilos e edeago, vista lateral; (5) estilos e conetivo, vista dorsal; (6) VII esternito da fêmea, vista ventral; (7) pigóforo da fêmea, vista lateral.

carenados e oblíquos. Fronte, em vista lateral, levemente arqueada; clípeo contínuo à fronte, de aspecto retangular, com margem apical retilínea. Pronoto mais largo que a cabeça, com comprimento mediano aproximadamente 1/3 da largura entre os úmeros; superfície dorsal finamente pontuada, margem posterior emarginada. Tégminas 3,4 vezes mais longas que largas; venação obscura, exceto apicalmente; com quatro células apicais, a base da terceira mais proximal que a da quarta. Pigóforo, em vista lateral, de forma triangular, três vezes mais longo do que sua largura basal, com margem posterior pontiaguda, terço apical com uma projeção triangular voltada para baixo, com microcerdas distribuídas apicalmente. Placa subgenital com aspecto mais ou menos retangular, inteiramente fundida, com o ápice mais estreito do que a base, margem posterior com reentrância mediana. Estilos longos, de comprimento quase igual ao da placa subgenital, com o ápice curvado para baixo, de aspecto unciforme. Conetivo em forma de "V" invertido, 
com os ramos delgados, sendo mais alargado na junção com o edeago. Edeago longo, subcilíndrico, largo basalmente, ápice curvado para baixo, com um processo basal voltado para a região anterior e dirigido ventralmente, ligando-se com o ápice do conetivo.

VII esternito da fêmea curto, mais ou menos retangular com uma reentrância mediana. Pigóforo feminino tão longo quanto largo, com margem apical truncada.

Coloração. No geral de cor amarela-pálida, apresentando manchas alaranjadas na cabeça e manchas marrons no escutelo.

Comentários. Coelidiana ferruginea sp.n. é semelhante às espécies do gênero, aproximando-se de C. durata Kramer, 1967 pelo aspecto da genitália do macho (pigóforo e edeago), porém difere por apresentar o ápice do edeago curvado para baixo.

\section{REFERÊNCIAS BIBLIOGRÁFICAS}

DeLong, D.M. 1953. A synopsis of the tribe Neocoelidiinii in the Americas. (Homoptera:Cicadellidae). Lloydia 16 (2): 93-131.

DeLong, D.M. \& A.B. Kolbe. 1975. Three new species of Neocoelidiinae (Homoptera: Cicadellidae) from Panama and Peru. Jour. Kansas Ent. Soc. 48 (1): 124-126.

Evans, J.W. 1947. A natural classification of leafhoppers (Jassoidea, Homoptera), Part 3: Jassidae.

Trans. ent. Soc. London 98 (6): 105-271.

Kramer, J.P. 1959. An elucidation of the Neotropical genus Chinaia with a key to males and a new allied genus (Homoptera: Cicadellidae: Neocoelidiinae). Proc. Biol. Soc. Wash. 72: 23-32.

-1964. A generic revision of the leafhopper subfamily Neocoelidiinae (Homoptera: Cicadellidae). Proc. U.S. Nat. Mus. 115 (3484): 259-288.

1967. New neotropical Neocoelidiinae with keys to the species of Coelidiana, Xenocoelidia, and Nelidina (Homoptera:Cicadellidae). Proc. Ent. Soc. Wash. 69 (1): 31-46.

LiNNAVUORI, R. 1965. On some new or interesting Neotropical Homoptera of the family Cicadellidae.

Zool. Beitr. 11 (1-2): 137-150.

Linnavuori, R. \& F. Heller. 1961. Beitrag zur Cicadelliden - Fauna von Peru. Stuttgarter Beitr. Naturk. 67: 1-14.

METCALF, Z.P. 1964. General catalogue of the Homoptera. Fascicle VI, Cicadelloidea. Bibliography of the Cicadelloidea (Homoptera: Auchenorrhyncha). Washington, D.C., Agricultural Research Service, U.S. Department of Agriculture, 349p.

OMAN, P.W. 1936. A generic revision of American Bythoscopinae and South American Jassinae. Univ.

Kansas Sci. Bull. 24 (16): 343-420.

Recebido em 31.III.1999; aceito em 18.III.2000

Revta bras. Zool. 17 (2): 539 - 542, 2000 